\title{
Investigation to predict patellar tendon reflex using motion analysis technique
}

\author{
L.K. Thama,*, N.A. Abu Osmana, K.S. Limb, B. Pingguan-Murphya, W.A.B. Wan Abasa, N. Mohd \\ Zaina
}

a Department of Biomedical Engineering, Faculty of Engineering, University of Malaya, Kuala Lumpur, Malaysia

b Division of Neurology, Faculty of Medicine, University of Malaya, Kuala Lumpur, Malaysia

Introduction

A reflex is an automatic and involuntary response to specific changes either outside or inside the body [1]. The functional unit of a reflex is the reflex arc, which is made up of a sensory organ, an afferent neuron, one or more synapses in the spinal cord, an efferent neuron, and an effector [2]. The deep tendon reflex can be obtained in a normal person as an immediate muscle contraction when muscle tendon is tapped briskly [3]. Medical practitioners commonly test the function of a human nervous system by eliciting reflexes [4].

Neuromuscular function can be assessed in general by performing reflex assessment. Any abnormality in the reflex response may indicate dysfunction in either the nervous system or the muscular system [5]. However, normal reflex assessments are observed visually by physicians, leading some times to unreliable judgments [6]. Such diagnoses lead to extremely subjective conclusions which may differ between examiners. The diagnostic results are therefore often inaccurate.

A new method is introduced by incorporating the motion analysis system into patellar tendon reflex assessment to obtain sets of accurate measurements for reflex responses which are diagnostically important. The motion analysis system, which involves optical tracking and video recording, provides quantitative data to predict reflexes accurately in amore objective way. This set of quantitative data is then compared between genders, different sides of the body and the effect of different striking forces.

\section{Methods}

The Queen square reflex hammer having a round rubber ring mounted on long plastic handle with sharpened end was chosen for the project due to its popularity among medical practitioners in the country. This type of reflex hammer has a longer handle and a heavier head than more common sizes of hammer, producing 
a greater swing force to the tendon and facilitating elicitation of

reflexes [7].

A reflex hammer holder was built to hold the reflex hammer at a

fixed position. The holder allows the height of the reflex hammer to

be adjusted according to the position of the patellar tendon (which

varies between subjects). The horizontal shaft of the holder was

modified from a commercial tile cutter and functions to clamp the

reflex hammer. Holes were drilled on both the cutter head and the end of the Queen square reflex hammer. The reflex hammer was

fixed on the tile cutter by a screw. The screw locking the reflex

hammer on the cutter restricts movement of reflex hammer to only

one plane. The vertical beam is the main structure supporting the

horizontal shaft and reflex hammer. Holes were drilled along the

length of the beam to enable the position of the reflex hammer to be

changed according to the knee position of different subjects. One

end of the beam is welded to a metal base to support the whole

structure.

Three levels of striking force were applied to the tendon during

experiments, enumerated Force 1, Force 2 and Force 3. Force 1

was intended to elicit hypo-reflexes, where the reflex hammer was

raised to an angle range up to 22 . In order to elicit a normal reflex,

the reflex hammer was raised to an angle in the range of $23-45^{\circ}$,

Force 2.

Force 3, meant for hyper-reflex is the greatest hammering

strength in which the reflex hammer is raised to an angle between

46. and 90 . The tapping force is derived from the potential energy

of the reflex hammer, which depends on the angle to which the

reflex hammer is raised.

The effect of the Jendrassik manoeuvre on patellar tendon reflex

was investigated in this project. The Jendrassik manoeuvre, a reinforcement

technique, is commonly applied in reflex tests when the

reflex response is not obvious for a particular subject [8]. When

applying the technique, the subject sat with the fingers interlocked

in front of the chest and attempted to pull the hands apart.

Fifty subjects comprising twenty eight males and twenty two

females, aged from 22 to 24 years participated in this project. All

subjects were healthy without any history of neurological disorders.

Subjects with neurological disease who might experience an

abnormal reflex response were excluded at recruitment. The male

subjects had an average height of $1.72 \pm 0.07 \mathrm{~m}$ and an average

body mass of $69.75 \pm 12.18 \mathrm{~kg}$. The female subjects had an average

height of $1.57 \pm 0.06$ mand an average body mass of $52.45 \pm 9.29 \mathrm{~kg}$. 
All subjects were selected in groups of similar Body Mass Index

(BMI) with an average BMI value of $22.01 \pm 1.72$ for males and

$20.32 \pm 2.18$ for female subjects.

The experimental setup for the project to tap on the patellar tendon for the collection of mean angle for the knee joints and the ankle joints is shown in Fig. 1.

As reported by the National Aeronautics and Space Administration (NASA, USA), the knee height of a male when sitting averages $52.6 \mathrm{~cm}$ [9]. Therefore, a stool with a height of $80 \mathrm{~cm}$ was prepared so that all subjects would sit with both legs hanging freely. Sixteen reflective markers were attached to the subject's lower limbs following the Plug-in-Gait Marker Placement [10]. During reflex tests, each subject was seated on a high stool with both legs hanging freely without touching the ground during the entire test. The patellar tendon on the right-hand side was tapped with the Queen square reflex hammer at Force 1 for 5 times. The same procedure was repeated at Force 2, Force 3 and using the Jendrassik manoeuvre. All tests were then repeated on the left patellar tendon. The reflex response was allowed to stop naturally before the next tap was applied. The tapping processes were recorded by the motion analysis system Nexus 1.3, where play back of the experiment allows detailed analysis. Kinematic data was then imported to another program, Vicon Polygon, for further analysis, where kinematic data was extracted in the form of quantitative values and graphs.

The jointmeanangles were statistically compared between genders and left and right legs using independent $t$ tests. Statistical significance was set at $\mathrm{P}<0.05$ for all tests. The effect of different striking forces and the Jendrassik manoeuvre was assessed using one-way analysis of variance (ANOVA). As post hoc analysis, Tukey's HSD test was carried out with $P<0.05$ for multiple comparisons. All data are represented as mean \pm standard deviation. 


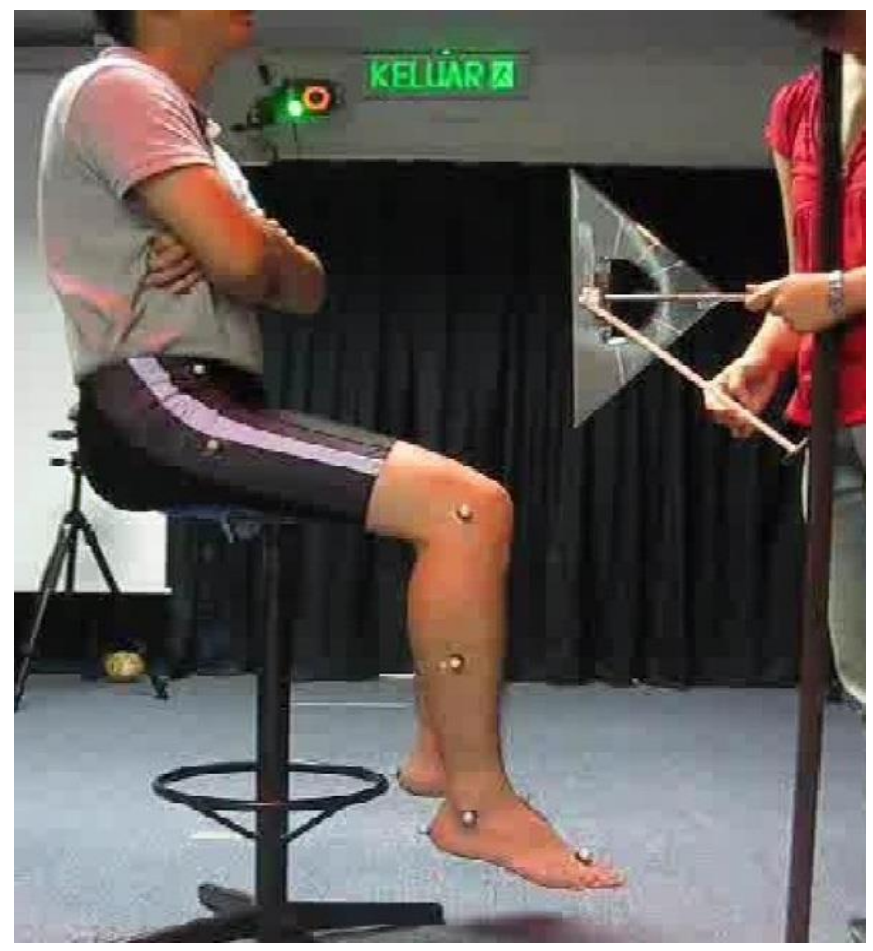

Fig. 1. Experimental setup for reflex assessment tests. The subject sits on a high stool with both feet not touching the ground. The patellar tendon of each leg is tapped using a Queen square reflex hammer. The Queen square reflex hammer is first raised to a certain angle measured using an attached protractor. The hammer is then released to tap on the patellar tendon. The experiment is recorded by the motion analysis system Nexus 1.3.

\section{Results}

Quantitative kinematic data for the patellar tendon reflex was collected and compared. Table 1 is the comparison of joint mean angle between genders. The mean angle for the knee joint and the ankle joint were labeled Angle 1, indicating flexion-extension for the knee, or dorsiflexion-plantarflexion for the ankle [11].

Angle 2 relates to adduction-abduction for the knee joint and inversion-eversion for the ankle joint [11]. Angle 3 refers to internal rotation or external rotation for the knee and ankle [11]. The reflex amplitude obtained shows that there was generally no statistical significance to the comparison of joint angles between males and females at different forces and the Jendrassik manoeuvre.

The mean angles for the knee joint and the ankle joint of both legs were then compared as represented in Table 2. Comparison of reflex responses between the left leg and the right leg shows that there were only slight differences in the reflex amplitude between the left leg and the right leg for all tapping conditions. These differences are not statistically significant except for knee angle 2 at force values 2 and the Jendrassik manoeuvre. 
Investigations were also performed for different striking forces

on reflex responses as represented in Table 3. The Jendrassik

manoeuvre was analyzed in order to observe the effect of reinforcement

on reflex response. Tapping at Force 2, equivalent to

the standard tapping angle for the normal knee reflex evaluation

method, was set as the control condition in the comparisons. Force

1 and Force 3, which aimed to elicit hypo and hyper reflexes in the

experiments, were being compared with the mean angles obtained

from Force 2. Tests on reflexes using the method of reinforcement

by the Jendrassik manoeuvre were done using a tapping force range

equal to Force 2 which is the normal reflex. Reflex amplitude for

the method used in the Jendrassik manoeuvre was again compared to the reflex amplitude obtained under normal reflex tests (Force

2).

Full text is available at :

http://ac.els-cdn.com/S1350453310002705/1-s2.0-S1350453310002705-main.pdf? tid=8773f9fe-a018-11e3-

83e4-00000aab0f26\&acdnat $=139355147659$ ec0bbc6ca1368b2d6cbac16e3cb683

http://www.ncbi.nlm.nih.gov/pubmed/21146440

http://www.sciencedirect.com/science/article/pii/S1350453310002705 